${ }^{4}$ Neonatal Unit, Royal Childrens Hospital; ${ }^{5}$ Department of Cardiology, Royal Children Hospital, Melbourne, VIC, Australia

Background Duct dependent congenital heart disease (DDCHD) may be difficult to distinguish from other diagnoses, notably persistent pulmonary hypertension (PPHN). Affected infants born in regional hospitals require transfer to a tertiary paediatric centre for echocardiographic diagnosis. Identification of predictive factors to distinguish DDCHD would assist in appropriate early management during transport.

Aims To describe transport of infants with suspected DDCHD and investigate early predictors of DDCHD.

Methods A retrospective study of infants with suspected DDCHD/PPHN transferred by NETS. Clinical, physiological and investigation data were compared between DDCHD and nonDDCHD (including PPHN) groups.

Results Of 142 eligible infants, 81 had DDCHD and 61 had nonDDCHD, of whom 51 had PPHN. There was no significant difference in median gestation, birth weight, sex, or distance of transfer between DDCHD and non-DDCHD groups. DDCHD infants were more often transferred by air than land.

At univariate analysis, presence of heart murmur, abnormal pulses, upper and lower limb BP difference $>10 \mathrm{mmHg}$, cardiomegaly, initial $\mathrm{SpO}_{2}$ of $<92 \%$ and pre-post ductal $\mathrm{SpO}_{2}$ difference $>10 \%$ were significantly associated with DDCHD. Labile $\mathrm{SpO}_{2}$, abnormal lung parenchyma, mean blood pressure $<40 \mathrm{mmHg}, \mathrm{pH}<7.25$, lactate $>5, \mathrm{PaO}_{2}<50 \mathrm{mmHg}$ and $\mathrm{FiO}_{2}>0.5$ were significantly associated with non-DDCHD.

On multivariate analysis, only labile $\mathrm{SpO}_{2}$ and mean BP $<40 \mathrm{mmHg}$ were significantly associated with non-DDCHD.

Conclusion Labile saturations and mean $\mathrm{BP}<40 \mathrm{mmHg}$ at the time of referral were significantly associated with non-DDCHD/PPHN. Other clinical features and physiological measures did not distinguish DDCHD from non-DDCHD.

\section{CARDIAC INVOLVEMENT IN NEWBORN OF DIABETIC MOTHER: DIAGNOSIS AND FOLLOW UP OF EVOLUTION}

doi:10.1136/archdischild-2012-302724.1144

'L Dimitriu, ${ }^{2}$ AG Dimitiru. 'Pediatric Cardiology, Medex Medical Center; ${ }^{2}$ Pediatric Cardiology, University of Medicine and Pharmacy, lasi, Romania

Objectives To present the main aspects of diagnosis and follow up of cardiac involvement in newborn of diabetic mothers (IDMs).

Methods/patients 84 IDMs investigated in the first week of life and re evaluated at 6-12 month (49), by clinical exam, ECG, cardiothoracic radiography (Rx.CT) and Doppler echocardiography (Echo). Fetal echo was performed in 38 cases.

Results Fetal Echo showed: cardiomegaly and myocardial hypertrophy of left ventricle (LV) (11 cases) confirmed by postnatal Echo. Clinical exam in newborn: macrosomia (40\%), systolic murmur (32 cases), signs of heart failure (3), others being asymptomatic or non cardiac symptoms. ECG: LV hypertrophy (14 cases). Rx.CT: cardiomegaly (12). Echo showed: non obstructive hypertrophic cardiomyopathy (HCMP) with asymmetric IVS hypertrophy (42\%), arterial pulmonary hypertension (6), LV diastolic dysfunction with normal systolic function (52\%) and congenital cardiac anomalies in $17 \%$ cases. HCMP was not correlated with the type of mother's diabetes, before pregnancy or gestational, but rather to an inadequate control of disease. Control performed at 6-12 months (21 cases): normal morphological cardiac aspect (14) or significant reduction of HCMP (7), with normal diastolic and systolic LV function.

Conclusions IDMs presents a high risk for cardiac involvement, either cardiac congenital malformations $(17 \%)$ or acquired cardiac pathology: HCMP (43\%) and disturbances of diastolic function of LV (54\%) and justify early cardiologic screening for all of these newborns with or without of cardiac suffering symptoms. Fetal and post natak Echo are the most sensitive and noninvasive methods of diagnostic and follow up on IDMs cardiac diseases.

\section{EFFECTS OF IBUPROFEN ON CEREBRAL AND SOMATIC REGIONAL TISSUE OXYGENATION, USING NIRS IN PRETERM INFANTS WITH A SIGNIFICANT PDA}

doi:10.1136/archdischild-2012-302724.1145

${ }^{1} \mathrm{~K}$ Gorman, 'RM Pinnamaneni, 1,20 Franklin, ${ }^{1,3} \mathrm{~A}$ Foran. ${ }^{1}$ Department of Paediatrics, Rotunda Maternity Hospital; '2Department of Cardiology, Our Lady's Children's Hospital Crumlin; ${ }^{3}$ Department of Neonatology, Childrens University Hospital Temple Street, Dublin, Ireland

Introduction To investigate the impact of a haemodynamically significant patent ductus arteriosus (PDA) and its treatment with ibuprofen on regional tissue oxygenation, both cerebral and somatic (gut) using near infrared spectroscopy (NIRS).

Method Prospective observational study in the neonatal intensive care unit of Rotunda Maternity Hospital from May 2011 to April 2012. Infants $<34$ weeks and $<1500$ g with a haemodynamically significant PDA confirmed on echocardiogram by Consultant Paediatric Cardiologist (OF) were eligible for the study. The machine used was the Somanetics INVOS system. NIRS sensors were applied to the head and liver for a minimum of 5 hours pre and 12 hours post treatment. Control patients were infants who met the above criteria but had a contraindication to ibuprofen therapy.

Results Total of 18 recordings for 15 infants, 10 in treatment group and 8 in control group. 2 infants received ibuprofen on 2 occasions. 9 male, 6 female. The mean gestational age was 26.63 (23.71-28.12) weeks and birth weight 798(550-1230)grams. The average recording was 13.46 hours pre-treatment, 39.78 hours post treatment and 39.97 hours in the control group. The median [range] cerebral regional oxygenation (cRO) pre-treatment was 68 [60-79] and post treatment was 70 [56.6-78.5]. The median [range] somatic regional oxygenation (sRO) pre-treatment was 50 [15-79] and post treatment was 54 [15-69.5]. In the control group, the median [range] cRO was 69.75 [62-78] and sRO was 40 [14-71.5].

Conclusion There was no statistically significant difference in regional tissue oxygenation (cerebral and somatic) pre and post treatment with ibuprofen.

\section{PERFUSION INDEX VARIABILITY AND N-TERMINAL PRO-BRAIN NATRIURETIC PEPTIDE LEVELS BEFORE AND AFTER CARDIAC INTERVENTIONS IN CONGENITAL HEART DISEASE}

doi:10.1136/archdischild-2012-302724.1146

'N Karadag, 'D Dilli, 'B Karagol, 'A Dursun, 'A Kundak, 'N Hakan, ${ }^{2} \mathrm{U}$ Arman Orun, ${ }^{1} S$ Beken, 'A Zenciroglu, 'N Okumus. 'Dr Sami Ulus Maternity, Childrens Education and Research Hospital, Division of Neonatology; ${ }^{2}$ Dr Sami Ulus Maternity, Childrens Education and Research Hospital, Division of Cardiology, Ankara, Turkey

Background and Aims Congenital heart diseases (CHD) are the most common life-threatening anomalies with significant morbidity and mortality in newborns. The aim of this study was to evaluate perfusion index variability (PI) and pro-Brain Natriuretic Peptide (NT-proBNP) levels before and after cardiac interventions.

Methods A prospective study was performed on hospitalized newborns diagnosed with $\mathrm{CHD}$. Oxygen saturation $\left(\mathrm{SaO}_{2}\right)$, $\mathrm{PI}$, heart rate and serum NT-proBNP levels were obtained before and $6^{\text {th }}$ hour after cardiac interventions (catheterization or surgery) in all patients, by using Masimo Rainbow SET Radica 17 Monitor (Masimo Corp., Irvine, CA, USA). Duration of mechanical ventilation, morbidity and mortality rates were documented.

Results Thirty-four CHD diagnosed newborns were included. Pulmonary atresia (20.6\%), aortic coarctation $(14.7 \%)$, and transposition of great arteries $(11.82 \%)$ are the most common diagnoses 
Median (IOR) birth weight and gestational age were 3250g (2450$4300)$ and 38weeks (38-42) respectively. Fifty-eight percent of newborns were male. Rapidly rising of oxygen saturations and PI values after cardiac interventions were observed in all patients $\left(\mathrm{SaO}_{2} \%\right.$, before: $79 \pm 6.6$, after: $87.9 \pm 2.9, \mathrm{p}=0.001$ ) (PI before: $0.4 \pm 0.1$, after: $1.1 \pm 0.2, p=0.001)$. A significant decrease in NT-proBNP levels (pg/ $\mathrm{mL}$ ) were seen after therapeutic interventions too(before: $1547 \pm 629$, after: $911 \pm 262, \mathrm{p}=0.001)$. Six patients $(17.6 \%)$ required surgical intervention. The median (IOR) day of mechanical ventilation was 7 days (2-21). Proven sepsis $(n=8.23 \%)$, chronic lung disease $(n=5.14 \%)$, pulmonary hemorrhage $(n=4, \% 11)$, and pneumothorax $(n=3.8 \%)$ are the most detected complications. Mortality rate was $20 \%(n=5)$.

Conclusion Peripheral tissues are sensitive to alterations in perfusion. PI monitoring of these tissues could be an early marker of hypoperfusion. PI has significantly improved in correlation with $\mathrm{SpO} 2$ after therapeutic interventions. Assessment of PI and NTproBNP values could be used by monitoring peripheral tissues in critically ill newborns with CHD.

\section{RENAL IMPAIRMENT BY INDOMETHACIN FOR PDA IN VLBW INFANTS}

doi:10.1136/archdischild-2012-302724.1147

${ }^{1} Y$ J Kim, ${ }^{1} \mathrm{JY}$ Lee, ${ }^{1} \mathrm{C}$ Kim, ${ }^{1}$ SH Shin, ${ }^{1} \mathrm{SH}$ Son, ${ }^{1} \mathrm{KY}$ Choi, ${ }^{2} \mathrm{JA}$ Lee, ${ }^{3} \mathrm{CW}$ Choi, ${ }^{1} \mathrm{EK}$ Kim, ${ }^{1} \mathrm{HS}$ Kim, ${ }^{3} \mathrm{BI}$ Kim, ' $\mathrm{JH}$ Choi. 'Department of Neonatology, Department of Pediatrics, Seoul National University Children's Hospital; ${ }^{2}$ Department of Neonatology, Department of Pediatrics, Seoul National University Boramae Hospital; ${ }^{3}$ Department of Neonatology, Department of Pediatrics, Seoul National University Bundang Hospital, Seoul, Republic of Korea

The Aims of our study were to identify the risk factors of using indomethain to very low birth weight infants (VLBWIs) during treatment of PDA.

A retrospective review was undertaken of 95 VLBWIs who were born between January, 2008 and December, 2009, at Seoul National University Hospital NICU. Of the 158 infants, 103 infants were treated with indomethacin and 8 were excluded because one's mother had azotemia and 7 patients died within the first week of life. Patients were classified by renal insufficiency (RI) and normal renal function (NRF) group. RI group was defined as having oliguria or elevation of serum creatinine level over from $1^{\text {st }}$ dose of indomethacin administration until 2 days after finishing the course.

Fourty-nine infants were RI group and 46 were NRF group. Administration duration was longer (2.5 \pm 2.0 days vs. $1.5 \pm 1.1$ days, $\mathrm{p}=0.007)$ and number of dosages $(5.1 \pm 2.8$ days vs. $4.0 \pm 2.2$ days, $\mathrm{p}=0.048)$ and cumulative dose were higher in RI group $(0.85 \pm 0.52$ $\mathrm{mg} / \mathrm{kg}$ vs. $0.64 \pm 0.44 \mathrm{mg} / \mathrm{kg} \mathrm{p}=0.040$ ). Most of the clinical characters were not different between groups but dopamine administration rate $(28.6 \%$ vs. $8.7 \% ; p=0.013)$ and serum potassium level before administration of indomethacin $(6.1 \pm 1.5 \mathrm{mEq} / \mathrm{L}$ vs. $5.1 \pm 1.6 \mathrm{mEq} / \mathrm{L}$ $\mathrm{p}=0.005$ ) were significantly elevated in RI group.

Hyperkalemia before administrating indomethacin and frequency/ dose of indomethacin are related to occurrences of RI during indomethacin administration. Therefore, renal function monitoring and combined drugs which can influence the renal function should be monitored during treatment of PDA.

\section{A COMPARISON OF TWO STRATEGIES FOR THE MANAGEMENT OF PATENT DUCTUS ARTERIOSUS (PDA)}

doi:10.1136/archdischild-2012-302724.1148

A Paweletz, K Woodger, D Gardiner, N Subhedar. Neonatal Intensive Care Unit, Liverpool Women's Hospital, Liverpool, UK

Background and Aims The management of PDA in extremely preterm infants is controversial and there is no agreed optimal approach. Strategies that are commonly used include prophylactic, early asymptomatic or late symptomatic therapy. We describe our experience in changing from prophylactic indomethacin to late symptomatic treatment with ibuprofen.

Methods We collected data on all babies admitted $<28$ weeks' gestation and/or $<1000 \mathrm{~g}$ from an electronic patient database. We compared PDA diagnosis management, demographics and clinical outcomes in two six month time periods: period 1 was when we used a prophylaxis strategy with indomethacin and period 2 when we changed to late symptomatic treatment with ibuprofen.

Results

Abstract 1148 Table 1 Patient characteristics and clinical outcomes

\begin{tabular}{llll}
\hline & Period 1 & Period 2 & p \\
\hline $\mathrm{N}$ & 37 & 44 & \\
Birth weight $(\mathrm{g})$ & $875(710-940)$ & $820(706-940)$ & 0.08 \\
Gestation $(w k)$ & $26(25-27)$ & $26(25-27)$ & 0.85 \\
Symptomatic PDA & $1335 \%)$ & $19(43 \%)$ & 0.46 \\
Late treatment of PDA with IBU/IND & $6(16 \%)$ & $15(34 \%)$ & 0.07 \\
Duct ligation & $3(8 \%)$ & $1(2 \%)$ & 0.32 \\
Severe IVH (grade 3/4) & $6(16 \%)$ & $8(18 \%)$ & 1.0 \\
Chronic lung diease at 36 weeks PMA & $24(65 \%)$ & $26(60 \%)$ & 0.59 \\
Death & $11(31 \%)$ & $13(30 \%)$ & 0.92 \\
\hline
\end{tabular}

Conclusions Changing from a strategy of indomethacin prophylaxis to selective, late symptomatic treatment of PDA with ibuprofen did not result in a significant increase in babies with severe IVH, chronic lung diasease or those needing duct ligation.

\section{TREAT THE PDA? COMPARISON OF THE OUTCOME IN TREATED AND RESTRICTIVE TREATED VLBW INFANTS}

doi:10.1136/archdischild-2012-302724.1149

${ }^{1} \mathrm{~K}$ Linnemann, ${ }^{1} \mathrm{C}$ Kriegsmann, ${ }^{1} \mathrm{R}$ Stenger, ${ }^{2} \mathrm{C}$ Fusch, ${ }^{1} \mathrm{M}$ Heckmann. ${ }^{1}$ Neonatology and Pediatric Intensive Care Unit, University of Greifswald, Greifswald, Germany; ${ }^{2}$ Neonatology, Mc Master University, Hamilton, ON, Canada

Background Management of PDA is discussed controversial. In 2008 we changed our regime from restrictive treatment of PDA to treatment of PDA.

Aim Comparing the outcome of VLBW-Infants with two therapeutic PDA regimes: A restrictive treated and $B$ treated PDA.

Method Historical cohort- study of VLBW infants born between 2005 and 2007 (group A: n=120; treatment of PDA only if weight gain was inadequate $(<10 \mathrm{~g} / \mathrm{kg} / \mathrm{d}$ in spite of optimized nutrition) and between 2008 and 2010 (group B: $n=126$; treatment of "hemodynamic significant" PDA, diagnosed on the basis of reverse diastolic flow in the Truncus coeliacus or renal arteries $<$ day 3). Other NICU guidelines were not changed but probiotics for NEC-prophylaxis started in 2011. Outcome measures: rate of NEC, BPD, IVH, neurological outcome (Laewen questionnaire).

Results In group A the rate of ductus closure was significantly lower than in group B (A: 19/53, B: 29/45; $\mathrm{p}<0.01)$. PDA rate in both groups did not differ significantly (A: 53/120; B: 45/126; $\mathrm{p}=0.67$ ). Surgical closure rate was $9 \%$ in both groups. The incidences of NEC, BPD and IVH were not different between groups. The results of the neurological outcome were not significantly different, but follow up reached only $60 \%$ so far.

Conclusions Changes in practice of PDA treatment in VLBW infants resulted in a $28 \%$ increase in frequency of medical or surgical closure of PDA without change in short or long term neonatal outcome. Further controlled randomized studies are needed to confirm our results on restrictive treatment of PDA.

1150 THE INCIDENCE AND RISK FACTORS OF THE CONGENITAL CARDIAC MALFORMATIONS IN NEWBORN

doi:10.1136/archdischild-2012-302724.1150 